\title{
Red supergiants in star-forming galaxies
}

\author{
Livia Origlia ${ }^{1}$ and Ernesto Oliva ${ }^{2}$ \\ ${ }^{1}$ Astronomical Observatory of Bologna, Italy \\ ${ }^{2}$ Astrophysical Observatory of Arcetri, Italy
}

\begin{abstract}
We present some results on the burst properties in star forming galaxies, using near infrared stellar features typical of red supergiants and Brackett nebular lines which trace the presence of luminous $\mathrm{H}$ II regions.
\end{abstract}

Near-IR spectroscopy of star forming galaxies allow to study both the 'H II phase', as traced by the nebular Brackett lines in emission, and the 'red supergiant phase', as traced by the stellar absorption features. Nebular hydrogen lines are a measure of the number of ionizing photons and represent powerful clocks to constrain the burst age and duration.

We examine NGC 1614 and NGC 7714, two well studied starburst galaxies with strong star formation activity in their central regions. They show the simultaneous presence of both Wolf-Rayet stars (Conti 1991), which trace a very young (a few Myr old) burst episode, and red supergiants (Oliva et al. 1995), which trace star formation episodes of 6-10 Myr up to a few hundreds Myr old.

If continuous star formation at constant rate occurred, we would expect to also see luminous $\mathrm{H}$ II regions traced by large Brackett line equivalent widths. This is not the case for NGC 1614 and NGC 7714, where the bulk of the 'H II phase' is exhausting and the infrared luminosity is dominated by the red supergiants.

Therefore, as a possible star formation scenario in these galaxies we propose two alternatives: a continuous star formation at a fast decreasing rate (on a time scale shorter than the typical WR lifetimes) or a strong burst episode occurred $\sim 10-100 \mathrm{Myr}$ ago, as traced by the red supergiants, and a more recent (occurred a few Myr ago), less massive burst as traced by the WR stars.

\section{References}

Conti, P.S., ApJ 377, 115

Oliva, E., Origlia, L., Kotilainen, J. K., Moorwood, A. 1995, A\&A 301, 55 